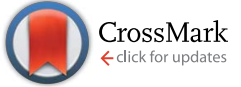

Cite this: RSC Adv., 2015, 5, 25929

\title{
Theoretical investigations of novel zinc oxide polytypes and in-depth study of their electronic properties $\dagger$
}

\author{
D. Zagorac, ${ }^{\star a b}$ J. C. Schön, ${ }^{a}$ J. Zagorac ${ }^{a b}$ and M. Jansen ${ }^{a}$
}

Zinc oxide is one of the most investigated compounds in materials science, both experimentally and theoretically, while in nature it appears only rarely, as the mineral zincite. Yet there are still many open questions: Is it still possible to observe or synthesize new modifications of zinc oxide? And can we improve the properties of a material that has already been investigated in thousands of studies? What is the connection between zincite, zinc sulfide and zinc oxide, and can we finally explain the controversial mineral matraite? In short, Yes: the answer to these questions is polytypism. We identify a multitude of possible stable polytypes for zinc oxide, and we show that by varying the stacking order, we can finetune the electronic properties such as the direct primary and secondary band gaps in zinc oxide without adding dopant atoms.

Received 17th December 2014

Accepted 4th March 2015

DOI: 10.1039/c4ra16574h

www.rsc.org/advances

should be capable of existence. Using global energy landscape

\section{Introduction}

In the literature, polytypism describes the phenomenon where a chemical compound crystallizes in a variety of periodically repeated layered structures - the polytypes. ${ }^{1}$ Usually, the individual layers constituting the polytypes are structurally identical, however the position of these layers relative to their neighboring layers can vary, leading to a multitude of stacking variants. Although quite a number of examples of compounds crystallizing in two or three such polytypes exist, ${ }^{2-6}$ it is not as common a phenomenon in experimental solid state chemistry as one would expect. There are still many open questions, e.g. why do some compounds show polytypism and others do not, how many polytypes can exist of one compound, and what is the effect of polytypism on the mechanical, thermal, or electronic properties?

One of the compounds where many polytypes are expected to exist is zinc oxide ( $\mathrm{ZnO})$, since the closely related zinc sulfide (ZnS) exhibits about 200 experimentally identified stacking variants. ${ }^{2}$ However, only three bulk phases of $\mathrm{ZnO}$ are known experimentally, a wurtzite and a sphalerite modification at ambient conditions, and a rocksalt phase at high pressures. ${ }^{7}$ As $\mathrm{ZnO}$ is a technologically very important compound, new modifications with different, possibly tunable properties would be highly desirable. Preliminary theoretical studies ${ }^{8}$ and subsequent work on nanofilms ${ }^{9}$ suggest that such polytypes

${ }^{a}$ Max Planck Institute for Solid State Research, Stuttgart, Germany

${ }^{b}$ Institute of Nuclear Sciences Vinča, Materials Science Laboratory, University of Belgrade,Belgrade, Serbia.E-mail: dzagorac@vinca.rs

$\uparrow$ Electronic supplementary information (ESI) available. See DOI: 10.1039/c4ra16574h exploration techniques, we have now identified a multitude of stable stacking variants of the wurtzite and the sphalerite type structure in zinc oxide, which are energetically nearly as low as the global minimum, the wurtzite modification.

In this study, we present the results of these global searches on the energy landscape of $\mathrm{ZnO}$ and analyze this plethora of newly discovered zinc oxide polytypes. We show that by varying the stacking order, we can fine-tune electronic properties such as the direct primary and secondary band gaps in zinc oxide.

Quite generally, all stable and metastable modifications of a chemical system correspond to the locally ergodic regions on the energy/enthalpy landscape of the system. At low or medium temperatures, such regions consist of local minima surrounded by sufficiently high energy barriers. ${ }^{10,11}$ As described in detail in the methods section, we proceeded in two steps during the global exploration of the energy landscape of zinc oxide: first, a number of local minima were identified using simulated annealing as a global optimization procedure, ${ }^{8,12}$ and in a second step, these minima served as starting points for further global explorations using the threshold algorithm. ${ }^{13,14}$ In the latter method, one performs random walks, usually starting from a local minimum, where every move is accepted as long as the new configuration remains below a given sequence of energy lids. Periodically, the random walker is quenched into the nearest local minimum, in order to find new local minima and to estimate the barriers separating them. Since we are interested in modifications that are stable at ambient conditions, we set the pressure to $p=0.1 \mathrm{MPa}$ for both the simulated annealing and the threshold runs (for simulated annealing based explorations of the enthalpy landscape of $\mathrm{ZnO}$ over a wide range of pressures, $c f$. ref. 12). 


\section{Computational methods}

Our general approach to the determination of structure candidates is based on the search for local minima and proceeds via the global exploration of the energy landscape of the system of interest. The method has been given in detail elsewhere; ${ }^{10,15}$ here, we just provide information specific to this study. The minima were identified using simulated annealing as a global optimization procedure. ${ }^{16}$ Since the global search involved many millions of energy evaluations, we employed an empirical energy/enthalpy function, $E=E_{\mathrm{pot}}+p V$, in this study. Here, $E_{\mathrm{pot}}$ $=\Sigma_{\langle i, j\rangle} V_{i j}\left(r_{i j}\right)$, where $V_{i j}$ is a two-body empirical potential consisting of Lennard-Jones and Coulomb terms:

$$
V_{i j}\left(r_{i j}\right)=\frac{q_{i} q_{j}}{4 \pi \varepsilon_{0} r_{i j}}+\varepsilon\left[\left(\frac{\sigma_{i j}}{r_{i j}}\right)^{12}-\left(\frac{\sigma_{i j}}{r_{i j}}\right)^{6}\right]
$$

Here, $q_{i}$ and $q_{j}$ are the charges of the ions, $r_{i j}$ is the distance between atoms $i$ and $j, \sigma_{i j}=r_{\mathrm{s}}(i) r_{\text {ion }}(i)+r_{\mathrm{s}}(j) r_{\text {ion }}(j)$ is the scaled sum of the ionic radii, and $\varepsilon$ gives the strength of the LennardJones potential. ${ }^{12}$ For the evaluation of the Coulomb summation, the method proposed by DeLeeuw was employed. ${ }^{17}$ Regarding the number of atoms in the simulation cell, we performed the global optimizations for 1, 2, 3, 4 and 5 formula units $\mathrm{ZnO}$ per simulation cell at standard pressure. Both the atom positions $(70 \%$ of all Monte Carlo steps move an individual atom, $10 \%$ swap the positions of two atoms) and the parameters of the periodically repeated simulation cell $(20 \%$ of all Monte Carlo steps) were freely varied during the random walks. Each simulated annealing run consisted of $4 \times 10^{6}$ Monte Carlo steps, followed by 10000 stochastic quench steps.

The barrier structure is explored using the threshold algorithm, ${ }^{14}$ where the landscape accessible from a local minimum below a sequence of energy barriers (thresholds) is systematically explored for all important local minima. In this study, we have again employed the two-body empirical potential consisting of Lennard-Jones and Coulomb terms, using up to 6 formula units. During these calculations the number of formula units $Z$ and the initial structure were varied. Each calculation consisted of 28 lids, ranging from -6.3 to $-3.9 \mathrm{eV}$, with a step size of $0.1 \mathrm{eV}$. In both cases, for the global exploration and the threshold algorithm calculations, we have used the modular G-42 code. ${ }^{10}$

Subsequent to the global search, the candidates found were locally optimized on ab initio level, and the $E(V)$ and $H(p)$ curves for the various modifications were computed, in order to be able to determine the thermodynamically stable ones as function of pressure. For this, we employed the program CRYSTAL09, ${ }^{18}$ based on linear combination of atomic orbitals (LCAO), and for the local optimizations we employed analytical gradients. ${ }^{19}$ Each local optimization was performed on the Hartree-Fock and the density functional theory (DFT) level, using the local density approximation (LDA). In addition we have employed a hybrid B3LYP functional (Becke's three-parameter functional in combination with the correlation functional of Lee, Yang, and $\left.\operatorname{Parr}^{20}\right)$. It is reasonable to use several different ab initio methods, in order to get some feeling for the quantitative validity of the results. ${ }^{21,22}$

For these local optimizations, all electron basis sets (AEBS) based on Gaussian-type orbitals (GTO) were used. In the case of $\mathrm{Zn}^{2+}$ a [6s5p2d] basis set was used as in ref. 23 and 24. For $\mathrm{O}^{2-}$ a [4s3p] basis set was used as in ref. 25 and 26 (see also ESI†े). During local optimizations a $k$-point sampling net of size $8 \times 8 \times 8$ was used. In addition, a smearing temperature of $0.01 E_{\mathrm{h}}$ was applied during the local optimization and the calculation of the $E(V)$ and $H(p)$ curves, in order to facilitate the numerical integration. For the analysis of the structures and their visualization we used KPLOT ${ }^{27}$ and the VESTA ${ }^{28}$ program.

\section{Results}

The global searches at standard pressure resulted in a plethora of local minima, which belong to a variety of structure types, such as the wurtzite, sphalerite, $5-5$, and $\beta$-BeO type (at high pressures, additional modifications were found, exhibiting e.g. the $\mathrm{NaCl}$, the NiAs, the GeP or the $\mathrm{CsCl}$ type $^{12}$ ). The appearance of these structure types was not surprising, since they also have been found on the energy landscape of a large number of other AB-systems such as the earth alkaline oxides ${ }^{29}$ or the alkali halides. ${ }^{30}$

However, during the threshold runs several modifications with very low energies comparable to the global minimum were found. These modifications correspond to stacking variants of the wurtzite and sphalerite structures, i.e., these modifications are the missing polytypes of zinc oxide (see Fig. 1). In order to confirm that these polytypes are not artefacts of the potential used, further simulated annealing runs using a Born-Mayertype potential (eqn (1)) were performed, yielding again these polytypes as local minima on the landscape. Furthermore, we note that when we have chosen one of the polytypes as starting minimum for the threshold runs, we required lid values of at least $0.5 \mathrm{eV}$ per atom, in order to leave this minimum basin on the energy landscape. Finally, the same periodic modification was observed with many different types of unit cells (while one often prefers to employ symmetry adapted unit cells for a structure description, there exist an infinite number of compatible unit cell of various sizes that can describe the same infinite periodic structure, many of which appear in landscape explorations when the simulation cell can be varied), again underlining the fact that these polytypes correspond to stable states on the energy landscape of $\mathrm{ZnO}$.

Regarding the $a b$ initio level, the local minimizations were performed both with and without symmetry prescriptions during the gradient-based minimization. Furthermore, we have changed the cell parameters and displaced the atoms from their apparent equilibrium values by finite amounts for all the minimum configurations, and repeated the local minimization procedure with three different $a b$ initio energy functions. In all cases, the structure returned to the stable position obtained originally. We note that this was true for both those polytypes that had appeared during the simulated annealing and threshold runs $(4 \mathrm{H}, 5 \mathrm{H}, 6 \mathrm{H}, 9 \mathrm{R})$ and those that had been constructed in analogy to the ZnS-system (8H, 12R, 15R). 


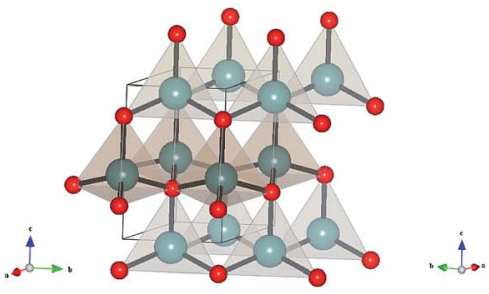

a)

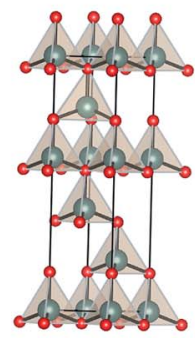

d)

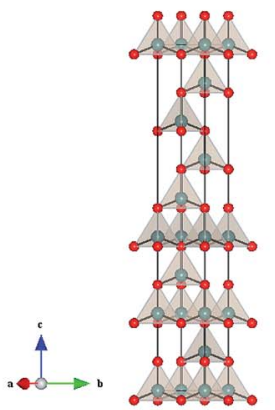

g)

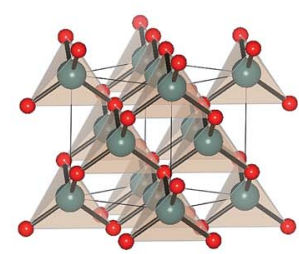

b)

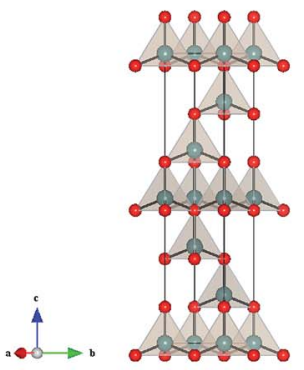

e)

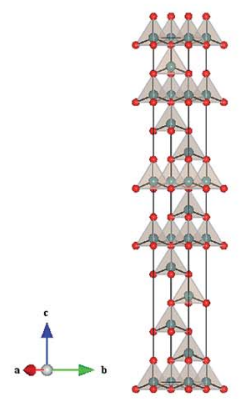

h)
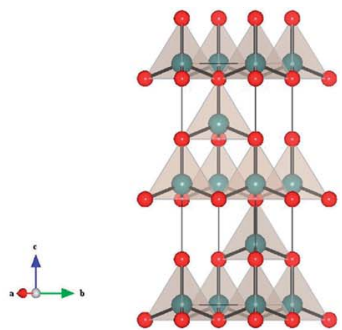

c)

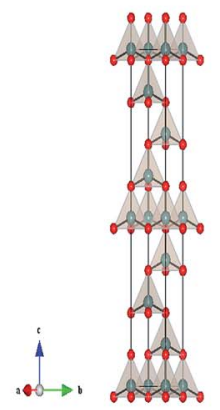

f)

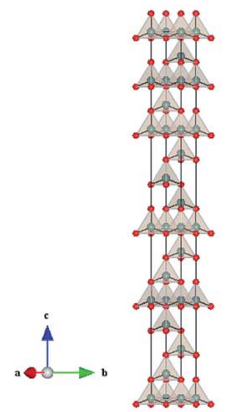

i)

Fig. 1 Visualization of the experimentally observed (a and b) and calculated (c-i) modifications of zinc oxide: (a) wurtzite (2H) type; (b) sphalerite (3C) type; (c) $4 \mathrm{H}$ polytype; (d) $5 \mathrm{H}$ polytype; (e) $6 \mathrm{H}$ polytype; (f) $8 \mathrm{H}$ polytype; (g) $9 \mathrm{R}$ polytype; (h) $12 \mathrm{R}$ polytype; (i) $15 \mathrm{R}$ polytype.

\subsection{Energetic ranking and structural features of the new polytypes}

The local optimizations of all the stable structure candidates on $a b$ initio level show that the wurtzite and sphalerite polymorphs of zinc oxide are the energetically lowest and thermodynamically most stable ones, which is in agreement with experimental observations $s^{7,31-34}$ and previous calculations. ${ }^{35-41}$ The wurtzite type together with the sphalerite type (see Fig. 1a) are the stable modifications at ambient conditions. For calculations performed on LDA and B3LYP level, these two minima have essentially the same energy, while on the Hartree-Fock level, their energies differ, $E_{\text {(wurtzite) }}=-1852.7170 E_{\mathrm{h}}<E_{\text {(sphalerite) }}=$ $-1852.7143 E_{\mathrm{h}}$ (see Table 1). Both modifications are well known in the zinc/iron sulfides with the analogous composition, ( $\mathrm{Zn}$, Fe)S. Wurtzite (B4) exhibits space group $\mathrm{Pb}_{3} \mathrm{mc}$ and its structure is composed of $\mathrm{ZnO}_{4}$ tetrahedra that are stacked in a hexagonal sequence $A B A B A B \ldots$ (hcp-packing). Sphalerite (B3) exhibits space group $F \overline{4} 3 m$, and its structure can be visualized as an $A B C A B C A B C \ldots$ cubic sequence of $\mathrm{ZnO}_{4}$ tetrahedra (fcc-packing). ${ }^{8}$
The appearance of the $4 \mathrm{H}, 5 \mathrm{H}, 6 \mathrm{H}$, and $9 \mathrm{R}$ stacking polytypes (see Fig. 1 and 2) in the global search and threshold runs is a clear indication that many other polytypes might be capable of existence in the $\mathrm{ZnO}$ system. The symbols used to classify the ZnO polytype structures have a specific meaning; e.g. the number 3 in the $3 \mathrm{C}$ polytype refers to the three layer periodicity of the stacking $(A B C)$ and the letter $C$ denotes the cubic symmetry of the crystal. The wurtzite $A B A B \ldots$ stacking sequence is denoted as $2 \mathrm{H}$, where 2 stands for two layer stacking periodicity and $\mathrm{H}$ for hexagonal symmetry. This periodicity doubles and triples in $4 \mathrm{H}$ and $6 \mathrm{H}$ polytypes. The family of rhombohedral polytypes is labelled with $R$, for example $9 R^{2}$ (see Fig. 1). Therefore, we performed a series of local optimizations on $a b$ initio level starting from other polytype structures known from ZnS: $8 \mathrm{H}, 12 \mathrm{R}$ and $15 \mathrm{R} .^{2,42}$ Of course, there exist many more hypothetical polytypes of $\mathrm{ZnS}$ and their stacking variants classified via the Zhdanov symbol; however, since their unit cells increase with the polytype number, we have not included them into the local ab initio energy minimizations. We would like to note a difference to the corresponding $\mathrm{ZnS}$ system, since there 
Table 1 Calculated total energies of the $\mathrm{ZnO}$ polytypes. Energies per formula unit are given in hartrees $\left(E_{\mathrm{h}}\right)$. Local optimizations were performed within the Hartree-Fock (HF), DFT (LDA), and hybrid functional (B3LYP) approximations. The temperature listed below the energy value is the energy difference to the wurtzite modification, suggesting thermodynamic stability at room temperature for the $4 \mathrm{H}, 5 \mathrm{H}, 6 \mathrm{H}, 8 \mathrm{H}$ and $12 \mathrm{R}$ polytypes, at least within the B3LYP approximation. Note that for the LDA-functional, the sphalerite modification is the lowest one in energy

\begin{tabular}{llll}
\hline & Energy $\left(E_{\mathrm{h}}\right)$ & & \\
\cline { 2 - 3 } & LDA & B3LYP & HF \\
\hline Structure type & -1851.2629 & -1854.5584 & -1852.7170 \\
Wurtzite $(2 \mathrm{H})$ & $(0 \mathrm{~K})$ & $(0 \mathrm{~K})$ & $(0 \mathrm{~K})$ \\
& -1851.2625 & -1854.5582 & -1852.7150 \\
$4 \mathrm{H}$ & $(+125 \mathrm{~K})$ & $(+60 \mathrm{~K})$ & $(+630 \mathrm{~K})$ \\
& -1851.2630 & -1854.5581 & -1852.7159 \\
$5 \mathrm{H}$ & $(-30 \mathrm{~K})$ & $(+90 \mathrm{~K})$ & $(+350 \mathrm{~K})$ \\
& -1851.2631 & -1854.5580 & -1852.7157 \\
$6 \mathrm{H}$ & $(-60 \mathrm{~K})$ & $(+125 \mathrm{~K})$ & $(+410 \mathrm{~K})$ \\
& -1851.2630 & -1854.5579 & -1852.7146 \\
$8 \mathrm{H}$ & $(-30 \mathrm{~K})$ & $(+160 \mathrm{~K})$ & $(+760 \mathrm{~K})$ \\
& -1851.2585 & -1854.5552 & -1852.7165 \\
9R & $(+1400 \mathrm{~K})$ & $(+1000 \mathrm{~K})$ & $(+160 \mathrm{~K})$ \\
& -1851.2627 & -1854.5578 & -1852.7153 \\
12R & $(+60 \mathrm{~K})$ & $(+190 \mathrm{~K})$ & $(+540 \mathrm{~K})$ \\
& -1851.2513 & -1854.5509 & -1852.7150 \\
15R & $(+3660 \mathrm{~K})$ & $(+2700 \mathrm{~K})$ & $(+630 \mathrm{~K})$ \\
& -1851.2632 & -1854.5575 & -1852.7143 \\
Sphalerite $(3 \mathrm{C})$ & $(-95 \mathrm{~K})$ & $(+285 \mathrm{~K})$ & $(+850 \mathrm{~K})$ \\
& & &
\end{tabular}

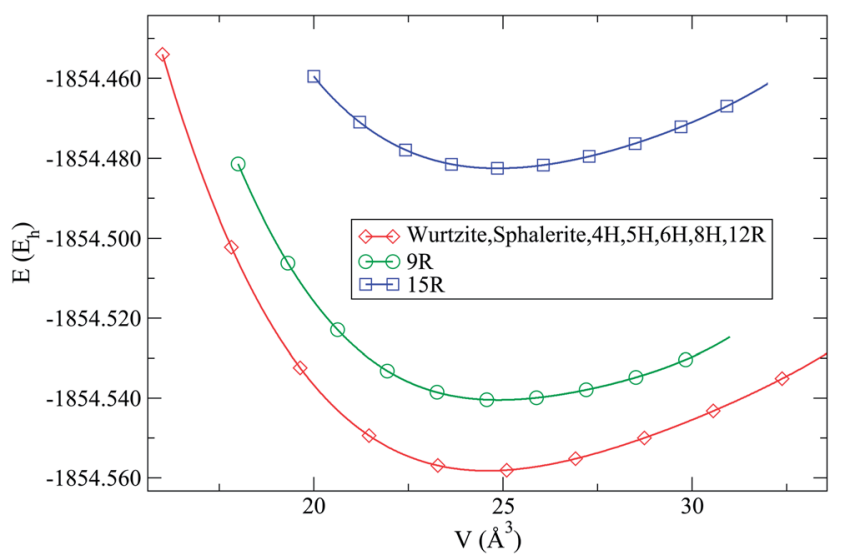

Fig. 2 Calculated $E(V)$ curves for the new stacking variants of $\mathrm{ZnO}$ at B3LYP level. Energies per formula unit are given in hartrees $\left(E_{h}\right)$. Note that most of the calculated $\mathrm{ZnO}$ polytypes $(4 \mathrm{H}, 5 \mathrm{H}, 6 \mathrm{H}, 8 \mathrm{H}$ and $12 \mathrm{R})$, as well as sphalerite structure, exhibit essentially the same $E(V)$ curve as wurtzite modification at this level of calculation and are indistinguishable on the scale of the figure, and therefore only the curve for wurtzite has been plotted.

exist no experimental or theoretical data of a $5 \mathrm{H}$ polytype in the $\mathrm{ZnS}$ system. On the other hand, the $5 \mathrm{H}$ polytype structure of ZnO that we have found in our global search and threshold runs, and that we have later optimized on ab intio level, shows very good agreement with the $5 \mathrm{H}$ polytype structure from the SiC system. ${ }^{43}$
Using DFT (LDA and B3LYP), we observe that several polytypes $(4 \mathrm{H}, 5 \mathrm{H}, 6 \mathrm{H}, 8 \mathrm{H}$ and $12 \mathrm{R})$ have nearly the same energy as the wurtzite $(2 \mathrm{H})$ and sphalerite (3C) modifications (see Fig. 2 and Table 1). Two polytypes were higher in energy at this level of calculations: the $9 \mathrm{R}$ and the $15 \mathrm{R}$ types. The $9 \mathrm{R}$ polytype was only slightly higher in energy at e.g. the B3LYP level $\left(E_{(9 \mathrm{R})}-E_{(2 \mathrm{H})}=\right.$ $0.0032 E_{\mathrm{h}}$, corresponding to about $1000 \mathrm{~K}$ ), but the $15 \mathrm{R}$ stacking variant could only be optimized as a distorted version, and was rather high in energy on DFT level.

Using Hartree-Fock (see Table 1), we observe that the $12 \mathrm{R}$ polytype is somewhat higher in energy, while the 9R polytype has an energy similar to the one of the wurtzite-type. Furthermore, the distorted $15 \mathrm{R}$ polytype, and also the $4 \mathrm{H}, 5 \mathrm{H}, 6 \mathrm{H}$ and $8 \mathrm{H}$ variants, are similar in energy to the sphalerite-type modification (see ESI†). A summary of structural data for each of the calculated $\mathrm{ZnO}$ polytypes including the full Zhdanov symbol is presented in the ESI. $\dagger$

\subsection{Electronic properties of the polytypes}

For the new predicted $\mathrm{ZnO}$ polytypes, we have performed band structure and density of states (DOS) calculations, in order to gain additional insight into the electronic properties of these possible modifications of zinc oxide. The calculations were performed using Hartree-Fock, DFT (LDA) and hybrid (B3LYP) functionals. Our calculations were in good agreement with previous experimental ${ }^{7,34,44,45}$ and theoretical findings, ${ }^{35,46-51}$ for those modifications where such data were available. Table 2 shows the band gaps calculated using different functionals for each of the $\mathrm{ZnO}$ polytypes investigated.

We can confirm the previous experimental and theoretical investigations of zinc oxide, where the primary direct band gap is observed at the $\Gamma$-point of the Brillouin zone. Sphalerite (3C), has the smallest gap of all the $\mathrm{ZnO}$ polytpes and wurtzite $(2 \mathrm{H})$

Table 2 Size of the calculated primary and secondary direct band gaps calculated with different $a b$ initio methods, of the ZnO polytypes. Note that the sphalerite modification has a Brillouin zone of different symmetry type, which cannot be described using the same symmetry directions as in the hexagonal polytypes

\begin{tabular}{|c|c|c|c|c|c|c|}
\hline \multirow[b]{2}{*}{ Structure type } & \multicolumn{3}{|c|}{$\begin{array}{l}\text { Primary direct band gap } \\
(\mathrm{eV})\end{array}$} & \multicolumn{3}{|c|}{$\begin{array}{l}\text { Secondary direct band } \\
\text { gap }(\mathrm{eV})\end{array}$} \\
\hline & LDA & B3LYP & $\mathrm{HF}$ & LDA & B3LYP & $\mathrm{HF}$ \\
\hline 2H (wurtzite) & 1.24 & 3.21 & 11.78 & 4.29 & 6.28 & 15.10 \\
\hline $4 \mathrm{H}$ & 1.20 & 3.15 & 11.67 & 2.37 & 4.25 & 12.59 \\
\hline $5 \mathrm{H}$ & 1.16 & 3.12 & 11.68 & 1.91 & 3.79 & 12.20 \\
\hline $6 \mathrm{H}$ & 1.17 & 3.11 & 11.58 & 1.79 & 3.66 & 12.11 \\
\hline $8 \mathrm{H}$ & 1.14 & 3.09 & 11.58 & 1.54 & 3.44 & 11.87 \\
\hline $9 \mathrm{R}^{a}$ & 1.19 & 3.16 & 11.66 & 2.13 & 4.04 & 12.48 \\
\hline $12 \mathrm{R}^{a}$ & 1.10 & 3.08 & 11.59 & 1.71 & 3.60 & 12.00 \\
\hline $15 \mathrm{R}^{a}$ & 1.14 & 3.13 & 11.57 & 1.59 & 3.48 & 11.91 \\
\hline 3C (sphalerite) & 1.12 & 3.08 & 11.57 & - & - & - \\
\hline
\end{tabular}

${ }^{a}$ The rhombohedral unit cell has been converted to a hexagonal unit cell for comparison. The secondary band gap occurs along the $\mathrm{H}-\mathrm{K}$ direction of the Brillouin zone, instead of at the $A$ point, as in those polytypes that are naturally occurring in the hexagonal unit cell. 
the largest one, computed with B3LYP and Hartree-Fock. Furthermore, an increase of the fraction of hcp-layers in the hexagonal polytypes (also known as hexagonality in the literature $^{52}$ ) usually results in a larger band gap.

In addition, a secondary band gap was observed for the first time in zinc oxide, and a direct correlation between the stacking order and the secondary band gaps is shown in Table 2. We observe similar trends of widening the secondary gap, as for the primary band gap; with the increase of hexagonality the band gap widens. This increase of the band gap is especially noticeable between the $2 \mathrm{H}$ and the $4 \mathrm{H}$ polytype, independently of the calculational method employed (see Table 2).

\section{Discussion}

In Fig. 3, we show the band structures of the $4 \mathrm{H}$ and $15 \mathrm{R}$ polytype calculated using the B3LYP functional. Both polytypes show a primary band gap at the $\Gamma$-point; however, the $4 \mathrm{H}$ polytype shows the secondary gap at the $A$ point, and the $15 \mathrm{R}$ polytype along the $\mathrm{H}-\mathrm{K}$ direction of the Brillouin zone. The complete set of band structures for ZnO polytypes calculated with various $a b$ initio methods are given in the ESI. $\dagger$

There have been many examples in various systems where different stacking orders, based on mixing the hcp- and the fcctype stacking, produce different polytypes. ${ }^{1-6}$ Our theoretical landscape explorations have also resulted in several interesting ZnO candidates which exhibit different stacking orders similar to the many polytypes in the $\mathrm{ZnS}$ system, while varying the numbers of formula units per simulation cell. Structurally, we can relate our polytype structures to those of zinc sulfide, or to the structures in silicon carbide, where different polytypes have also been observed..$^{2-4,42,43,53,54}$

Concerning the effects of polytypism on the electronic structure, it is known that e.g. in silicon carbide (SiC) the $3 \mathrm{C}$ polytype shows the highest electron mobility. ${ }^{4,55}$ Furthermore, the band gaps of SiC differ widely among the polytypes ranging from $2.3 \mathrm{eV}$ for the cubic $3 \mathrm{C}$ polytype (i.e. sphalerite) to $3.3 \mathrm{eV}$ for the hexagonal $2 \mathrm{H}$ polytype (i.e. wurtzite), where the experimental polytpyes investigated have band gaps in-between (e.g. the $6 \mathrm{H}$ polytype has a gap of $3 \mathrm{eV}$ ). But quite generally, one finds that the larger the fraction of wurtzite-like components $(2 \mathrm{H})$, the larger the band gap.,55 Our results show the same overall trends for the zinc oxide polytypes as in silicon carbide: sphalerite (3C) has the smallest gap of all the $\mathrm{ZnO}$ polytpes and wurtzite $(2 \mathrm{H})$ the largest, respectively, and by increasing the size of hcp layer regions one increases the band gap. Therefore, it should be possible, by combining fcc-type and hcp-type layer stackings, to reduce the size of the band gap (see Table 2). Note that in $\mathrm{ZnO}$ the $12 \mathrm{R}$ and $15 \mathrm{R}$ structures differ slightly from the $12 \mathrm{R}$ and $15 \mathrm{R}$ polytypes in $\mathrm{ZnS}$, respectively, and this may also influence the band gap (for the structure data, $c f$. ESI $\dagger$ ). Similar trends in the band gaps are observed in other III-V compounds. ${ }^{5}$

Clearly, the electronic effects of changing the stacking order in zinc oxide are not as dramatic as in silicon carbide. Nevertheless, this indicates that it is possible to tune the direct primary and secondary band gaps in $\mathrm{ZnO}$ to values between those of the wurtzite- and the sphalerite-modification by selecting the appropriate polytype without adding dopant atoms. Of course, the existence of polytypes makes it nontrivial to synthesize a macroscopic single-crystal; however if one can establish a degree of control during crystal growth sufficient to synthesize specific different polytypes, new materials for electronic device applications would be accessible. ${ }^{4,5,55}$ Recent attempts to generate nanofilms with unusual stacking orders ${ }^{9}$ are quite encouraging in this respect.

We note that the structure chemistry of zinc oxide and zinc sulfide appear to be quite similar. This similarity is demonstrated not only by the polytype calculations presented here, but also by many experimental observations, such as the existence of wurtzite and sphalerite modifications in both $\mathrm{ZnO}$ and $\mathrm{ZnS}$, and recent investigations that suggest the occurrence of calculated, ${ }^{56}$ synthetic $^{57,58}$ and natural ${ }^{59}$ polymorph of $\mathrm{ZnO}$ intergrown with ZnS. Therefore, we would like to comment on an ongoing discussion regarding the structural classification of the zinc sulfide mineral matraite, which has been claimed to be a 3R-polytype of ZnS. The most common polytypes of zinc sulfide are $2 \mathrm{H}$ (wurtzite) and $3 \mathrm{C}$ (sphalerite), while others are rarely encountered in nature..$^{2,54,60}$ The purported 3R polytype was for the first time experimentally synthesized in the U.S. almost

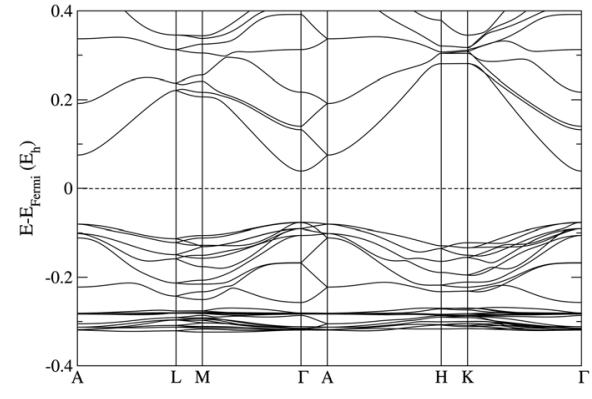

a)

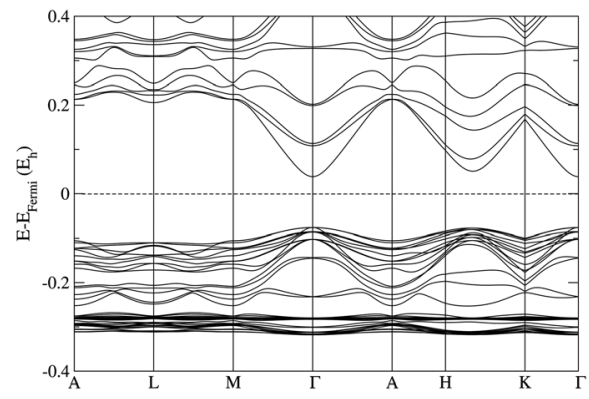

b)

Fig. 3 Band structure calculation using the hybrid B3LYP functional for (a) the 4H polytype; (b) the 15R polytype. Note that the labels of the special points of the Brillouin zones correspond to those of a hexagonal lattice. The rhombohedral unit cell is converted to a hexagonal one for comparison. 
sixty years ago, ${ }^{61}$ while its natural form as the mineral matraite has been found in Hungary, ${ }^{62}$ and later in Bulgaria. ${ }^{63}$ However, as alternatives, a $6 \mathrm{H}$ polytype ${ }^{60}$ or a twinned sphalerite structure have been suggested ${ }^{42,59,64}$ as possible models for the matraite structure. Now, if such a 3R-structure modification were to exist as a distinct polytype, we would expect to observe it also in $\mathrm{ZnO}$ during our nearly exhaustive threshold investigations. This not being the case, we have constructed the 3R-type according to the description in ref. 61-63, and compared this structure with the ones found during the global searches. This structure comparison using the CMPZ algorithm ${ }^{65}$ clearly shows that the $3 \mathrm{R}$ polytype is equal to the $3 \mathrm{C}$ polytype both in $\mathrm{ZnO}$ and in $\mathrm{ZnS}$, which would agree with the experimental results in ref. 42, 59 and 64 . In addition, the structural-crystallographic constraints in the $\mathrm{ZnO} / \mathrm{ZnS}$ compounds (or any analogous $\mathrm{AB}$ compound) show that the distances between the layers are the same as in the fcc packing, and therefore $3 \mathrm{C}$ must be equal to $3 \mathrm{R}$. An exception would only be possible, if some special feature of the electronic structure of zinc oxide or zinc sulfide were to favor a second (structurally anisotropic) energy minimum right next to the sphalerite structure minimum on the energy landscape. None of our ab initio calculations suggest the existence of such a double minimum basin. While recent work on elemental zinc ${ }^{66}$ has shown that zinc can exhibit several electronic minimum energy configurations which induce slightly different atom positions, the bonding situation in zinc oxide/sulfide is completely different, and the presence of electron correlations strong enough to generate a second minimum is very unlikely. This is the reason, why in our global searches we observe only the $3 \mathrm{C}$ and $9 \mathrm{R}$ polytypes but not a separate $3 \mathrm{R}$ polytype (see Fig. 1). We would thus conclude that the matraite mineral is not a separate modification, but rather a (possibly twinned) 3C (sphalerite) polytype of $\mathrm{ZnS}$.

\section{Conclusions}

To summarize, in order to gain new insights into the polytypism of the $\mathrm{ZnO}$ compound, we have performed structure prediction using simulated annealing and threshold runs with an empirical potential, followed by local minimizations on $a b$ initio level. We have found the experimentally known structure types (wurtzite, sphalerite and rock-salt), in agreement with previous research, and we observe new low-energy polytypes of zinc oxide, which should be accessible to the experiment. A second focus of this research has been the investigation of the electronic properties of the newly discovered polytypes. We observe the same trends in the values of the band gap as in silicon carbide and zinc sulfide, and we note that the secondary band gap plays a role in zinc oxide polytypes. Clearly, these results suggest important new avenues in the development and fine-tuning of electronic devices with possible industrial applications. Additionally, we have clarified the ongoing discussion regarding the matraite (3R) mineral in zinc sulfide, which we can describe as a (possibly twinned) sphalerite (3C) structure.

\section{Acknowledgements}

The authors would like to thank A. Recnik, J. Nuss, K. Doll, B. Matovic, K. Djuris and U. Wedig for valuable discussions.

\section{References}

1 U. Müller, Inorganic Structural Chemistry, Wiley-VCH, Marburg, Germany, 2007.

2 S. Mardix, Phys. Rev. B: Condens. Matter Mater. Phys., 1986, 33, 8677.

3 J.-S. Lee, S.-C. Yu, S.-F. Tung, W.-J. Bai, J.-S. Yang, Q.-S. Fang and Z. Zhang, Z. Kristallogr., 2006, 221, 213.

4 H. Morkoc, S. Strite, G. B. Gao, M. E. Lin, B. Sverdlov and M. Burns, J. Appl. Phys., 1994, 76, 1363.

5 F. Bechstedt and A. Belabbes, J. Phys.: Condens. Matter, 2011, 25, 273201.

6 S. Nakashima, H. Katahama, Y. Nakakura, A. Mitsuishi and B. Palosz, Phys. Rev. B: Condens. Matter Mater. Phys., 1985, 31, 6531.

7 Ü. Özgür, Ya. I. Alivov, C. Liu, A. Teke, M. A. Reshchikov, S. Doğan, V. Avrutin, S.-J. Cho and H. Morkoç, J. Appl. Phys., 2005, 98, 041301.

8 D. Zagorac, J. C. Schön, I. Pentin and M. Jansen, Process. Appl. Ceram., 2011, 5, 73.

9 M. Liu, G.-B. Ma, X. Xiong, Z.-W. Wang, Ru-W. Peng, J.-G. Zheng, Da-J. Shu, Z. Zhang and M. Wang, Phys. Rev. B: Condens. Matter Mater. Phys., 2013, 87, 085306.

10 J. C. Schön and M. Jansen, Angew. Chem., Int. Ed. Engl., 1996, 35, 1268.

11 S. M. Woodley and C. R. A. Catlow, Nat. Mater., 2008, 7, 937.

12 D. Zagorac, J. C. Schön, J. Zagorac and M. Jansen, Phys. Rev.

B: Condens. Matter Mater. Phys., 2014, 89, 075201.

13 D. Zagorac, J. C. Schön, J. Zagorac, I. V. Pentin and M. Jansen, Process. Appl. Ceram., 2013, 7, 111.

14 J. C. Schön, H. Putz and M. Jansen, J. Phys.: Condens. Matter, 1996, 8, 143.

15 J. C. Schön, K. Doll and M. Jansen, Phys. Status Solidi B, 2010, 247, 23.

16 S. Kirkpatrick, C. D. Gelatt and M. P. Vecchi, Science, 1983, 220, 671.

17 S. W. de Leeuw, J. W. Perram and E. R. Smith, Proc. R. Soc. London, Ser. A, 1980, 373, 27.

18 R. Dovesi, R. Orlando, B. Civalleri, C. Roetti, V. R. Saunders and C. M. Zicovich-Wilson, Z. Kristallogr., 2005, 220, 571.

19 K. Doll, R. Dovesi and R. Orlando, Theor. Chem. Acc., 2004, 112, 394.

20 A. Becke, J. Chem. Phys., 1993, 98, 5648.

21 J. C. Schön, Z. P. Cancarevic and M. Jansen, J. Chem. Phys., 2004, 121, 2289.

22 D. Zagorac, K. Doll, J. C. Schön and M. Jansen, Phys. Rev. B: Condens. Matter Mater. Phys., 2011, 84, 045206.

23 J. E. Jaffe and A. C. Hess, Phys. Rev. B: Condens. Matter Mater. Phys., 1993, 48, 7903.

24 T. Homann, U. Hotje, M. Binnewies, A. Borger, K. D. Becker and T. Bredow, Solid State Sci., 2006, 8, 44. 
25 M. D. Towler, N. L. Allan, N. M. Harrison, V. R. Saunders, W. C. Mackrodt and E. Apra, Phys. Rev. B: Condens. Matter Mater. Phys., 1994, 50, 5041.

26 A. M. Ferrari and C. Pisani, J. Phys. Chem., 2006, 110, 7909.

27 R. Hundt, KPLOT program-Version 9.6.15, University of Bonn, Bonn, Germany, 2012.

28 K. Momma and F. Izumi, J. Appl. Crystallogr., 2008, 41, 653. 29 J. C. Schön, Z. Anorg. Allg. Chem., 2004, 630, 2354.

30 Z. Cancarevic, J. C. Schön and M. Jansen, Chem.-Asian J., 2008, 3, 561.

31 C. H. Bates, W. B. White and R. Roy, Science, 1962, 137, 993.

32 H. Liu, Y. Ding, M. Somayazulu, J. Qian, J. Shu, D. Häuserman and H.-K. Mao, Phys. Rev. B: Condens. Matter Mater. Phys., 2005, 71, 212103.

33 F. Decremps, J. Zhang, B. Li and R. C. Liebermann, Phys. Rev. B: Condens. Matter Mater. Phys., 2001, 63, 224105.

34 S.-K. Kim, S.-Y. Jeong and C. R. Cho, Appl. Phys. Lett., 2003, 82, 562 .

35 J. E. Jaffe, J. A. Snyder, Z. Lin and A. C. Hess, Phys. Rev. B: Condens. Matter Mater. Phys., 2000, 62, 1660.

36 D. Zagorac, J. C. Schön and M. Jansen, J. Phys. Chem. C, 2012, 116, 16726.

37 C. R. A. Catlow, S. A. French, A. A. Sokol, A. A. Al- Sunaidi and S. M. Woodley, J. Comput. Chem., 2008, 29, 2234.

38 A. Kawska, P. Duchstein, O. Hochrein and D. Zahn, Nano Lett., 2008, 8, 2336.

39 M. Hellström, K. Jorner, M. Bryngelsson, S. E. Huber, J. Kullgren, T. Frauenheim and P. Broqvist, J. Phys. Chem. C, 2013, 117, 17004 .

40 M. R. Farrow, Y. Chow and S. M. Woodley, Phys. Chem. Chem. Phys., 2014, 16, 21119.

41 B. Meyer and D. Marx, Phys. Rev. B: Condens. Matter Mater. Phys., 2003, 67, 035403.

42 S. Haussuehl and G. Müller, Beitr. Mineral. Petrogr., 1963, 9, 28.

43 R. G. Gevorkyan, G. A. Gurkina and F. V. Kaminskii, Am. Mineral., 1976, 61, 1054.

44 D. Milivojevic, B. Babic-Stojic and J. Kovac, Appl. Surf. Sci., 2010, 257, 937.

45 K. Siraj, K. Javaid, J. D. Pedarnig, M. A. Bodea and S. Naseem, J. Alloys Compd., 2013, 563, 280.
46 Z. Zeng, C. S. Garoufalis, S. Baskoutas and G. Bester, Phys. Rev. B: Condens. Matter Mater. Phys., 2013, 87, 125302.

47 J. Wrobel and J. Piechota, Solid State Commun., 2008, 146, 324.

48 Z. Li, Y. Xu, G. Gao, T. Cui and Y. Ma, Phys. Rev. B: Condens. Matter Mater. Phys., 2009, 79, 193201.

49 S. H. Jang and S. F. Chichibu, J. Appl. Phys., 2012, 112, 073503.

50 I. Demiroglu, S. Tosoni, F. Illasa and S. T. Bromley, Nanoscale, 2014, 6, 1181.

51 L. Sponza, J. Goniakowski and C. Noguera, Phys. Rev. B: Condens. Matter Mater. Phys., 2015, 91, 075126.

52 K. Dornberger-Schiff and H. Schmittler, Acta Crystallogr., Sect. A: Cryst. Phys., Diffr., Theor. Gen. Crystallogr., 1971, 27, 216.

53 H. Schulz and K. H. Thiemann, Solid State Commun., 1979, 32, 783.

54 G. Y. Chao and R. A. Gault, Can. Mineral., 1998, 36, 775.

55 M. Bhatnagar and B. J. Baliga, IEEE Trans. Electron Devices, 1993, 40, 645.

56 J. Goclon and B. Meyer, Phys. Chem. Chem. Phys., 2013, 15, 8373.

57 M.-Y. Lu, J. Song, M.-P. Lu, C.-Y. Lee, L.-Y. Chen and Z. L. Wang, ACS Nano, 2009, 3, 357.

58 G. Homm, et al., J. Electron. Mater., 2010, 39, 1504.

59 V. Srot, A. Recnik, C. Scheu, S. Sturm and B. Mirtic, Am. Mineral., 2003, 88, 1809.

60 M. Pósfai and P. R. Buseck, Modular structures in sulphides: sphalerite/wurtzite-, pyrite/marcasite-, and pyrrhotite-type minerals, in EMU Notes in Mineralogy: Modular Aspects of Minerals, ed. S. Merlino, European Mineralogical Union (EMU), Budapest, Hungary, 1997, vol. 1, pp. 193-235.

61 D. C. Buck and L. W. Strock, Am. Mineral., 1955, 40, 192.

62 S. Koch, Acta Mineral.-Petrogr., 1958, 11, 11.

63 J. A. Minceva-Stefanova, Mineral. Petrol., 1993, 49, 119.

64 E. Nitta, et al., J. Mineral. Petrol. Sci., 2008, 103, 145.

65 R. Hundt, J. C. Schön and M. Jansen, J. Appl. Crystallogr., 2006, 39, 6 .

66 N. Gaston, D. Andrae, U. Wedig and M. Jansen, Phys. Rev. Lett., 2008, 100, 226404. 